Recibido: 06-06-2016

Aceptado: 25-06-2016

Palabras clave: memoria semántica, jóvenes, camu camu, maca negra, antioxidante, hormonas, rendimiento fisico.

Key words: Semantic memory, camu camu, maca, black maca, young people, antioxidant, hormone.

Geraldine Salazar geraldine.salazar@upch.pe UPCH

Carmen Estela Montenegro Serkovic carmen.montenegro@upch.pe UPCH

Gustavo Gonzales UPCH

Luis Aguilar UPCH - UNIFE luis.aguilar@upch.pe

\section{Estudio de la memoria semántica en estudiantes de una universidad de la Amazonía del Perú, luego del consumo de Camu Camu y Maca Negra}

\section{A study on semantic memory in students who have eaten camu camu and black maca of a university in the Amazon region of Peru}

Salazar, Geraldine'; Montenegro, C. Estela'; Gonzales, Gustavo² Aguilar, Luis ${ }^{3}$

RESUMEN. En el presente estudio, que es parte de un Proyecto de Investigación más amplio denominado "Evaluación Metabólica y efecto Antioxidante de Camu Camu y Maca como nutracéutico en jóvenes de 18 a 25 años de la Amazonia Peruana", se evaluaron el efecto cognitivo de dos plantas, maca negra (Lepidium meeenii) y camu camu (Myrciaria dubia), en la memoria semántica y procedimental de estudiantes universitarios de la Universidad Nacional de la Amazonia. La pulpa de camu camu y extracto de maca negra atomizados fueron suministrados vía oral a 80 estudiantes entre 18 a 25 años, divididos en 4 grupos. Cada grupo recibio durante 6 semanas las sustancias según la siguiente distribución, Grupo 1: Maltodextrina, Grupo 2: Camu camu, Grupo 3: Maca negra, Grupo 4: Maca negra y Camu camu. Los efectos en la memoria semantica se evaluo al inicio y al final del ensayo, utilizando la prueba Neuropsicológica: "Palabras de Rey para la memoria Semántica".

Los datos fueron ingresados en una base de datos en el programa EXCEL y luego fueron transferidos al programa STATA versión 12 donde se hicieron los análisis estadísticos.

Los resultados muestran un significativo incremento de la memoria semántica como efecto de la ingesta de maca negra y camu camu, solo o combinado, siendo este incremento mayor con la maca negra.

ABSTRACT. The study is of a broader research project called "Metabolic evaluation and anti-oxidative effect of Camu Camu and Maca as a nutraceutical in young people aged 18 to 25 in the Peruvian Amazon University". This proyect evaluated the cognitive effect of two plants, black maca (Lepidium meyenii) and camu camu (Myrciaria dubia), in the semantic and procedural memory of college students from the National University of the Amazon. The pulp of camu camu and extract of black maca atomized were delivered orally to 80 students between 18-25 years, divided into 4 groups. Each group received 6 weeks according to the following distribution, group 1 substances: maltodextrin, group 2: Camu camu, group 3: black Maca, group 4: black Maca and Camu camu. The effects in semantic memory is assessed at the beginning and at the end of the trial, using the Neuropsychological Test: "The Rey words for semantic memory".

The statistical analyses used the database in EXCEL program and it was transferred to the STATA program 12.

The results show a significant increase of semantic memory as an effect of the intake of black maca and camu camu, alone or in combination, being this higher with black maca increase. 


\section{I.- INTRODUCCIÓN}

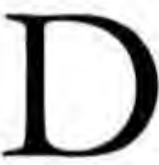

ebido a los efectos benéficos del camu camu y la maca, este último considerado como producto de bandera peruano, nace la iniciativa de realizar un estudio en jóvenes universitarios aparentemente normales para determinar el efecto de la ingesta de dichos productos, evaluando el beneficio en la memoria.

Las propiedades antioxidantes del camu camu (Myrciaria dubia), se han demostrado en diversos estudios en los últimos 15 años (Villanueva-Tiburcio et. al. 2010, Chirinos et. al. 2010, Sotero et al 2009, Muñoz et al, 2009, Ramos et al 2008; Klinar et al 2009). Este fruto amazónico es reconocido por su alta concentración de ácido ascórbico (Collazos et al,1957; Bradfield et al,1964; Yuyama et al, 2002), las propiedades antioxidantes no solo se deben al elevado contenido de vitamina C (Justi et al, 2000; Inoue et al, 2008) sino también a otras sustancias algunas identificadas y otras aún desconocidas (Villanueva-Tiburcio et al, 2010; Muñoz et al, 2009, 2007; Zanatta et al, 2005, 2007; Reynertson et al, 2008; Genovese et al, 2008; Sotero et al, 2009) .

La maca (Lepidium meyenii) es una planta altoandina con propiedades que afectan algunas funciones cognitivas (Pino-Figueroa et al, 2010; Rubio et al, 2007). Esta planta también afecta algunas funciones sistémicas como la osteoporosis (Gonzales et al, 2010; Wang et al, 2009), la fertilidad el deseo sexual y la actividad física (Stone et al, 2009; Zenico, 2009. Estas propiedades, entre otras, colocan a esta planta con un alto valor potencial como alimento, que puede ser aprovechado no solo para combatir las enfermedades sino también la malnutrición y desnutrición infantil junto a las secuelas de las mismas.

1. A nivel Conceptual consideramos la relevancia del estudio de la memoria, que en los seres humanos hace posible, además de consolidar la experiencia como una continuación del pasado, el poder tener una proyección hacia el futuro, es debido a ello que la memoria es la base de todo nuestro conocimiento, habilidades, planes y proyectos (Prado, R. y Qiuirarte , G. 1998).

2. Nutracéuticos, hacen referencia a todos aquellos alimentos que se proclaman como poseedores de un efecto beneficioso sobre la salud humana, como la Maca y el Camu Camu. Del mismo modo, el término puede aplicarse a compuestos químicos individuales presentes en comidas comunes como algunos fitoquímicos. El término fue acuñado en 1989 por el Dr. Stephen De Felice.

\section{II.- OBJETIVOS}

Objetivo General, evaluar el efecto del consumo de los extractos atomizados de camu camu y maca negra, solos o en combinación en la memoria semántica en jóvenes de 18 a 25 años de la Universidad de la Amazonía Peruana.

\section{Objetivos Específicos}

(1) Evaluar el efecto simple del camu camu, y maca negra, solos, sobre la memoria semántica a través de la prueba de Palabras de Rey. (2) Evaluar el efecto del camu camu y maca negra combinados, sobre la memoria. (3) Comparar la curva de aprendizaje generado por la prueba Palabras de rey entre el grupo que consumió camu camu y maca negra solos o en combinación. (4) Comparar por sexo el efecto del consumo de camu camu y maca negra, solos o combinados, sobre la memoria semántica. (5) Comparar por edad el efecto del consumo de camu camu y maca negra, solos o combinados, sobre la memoria semántica.

\section{III.- MATERIAL Y MÉTODOS}

Instrumento: Se aplicó el Test de Palabras de Rey, Memoria Auditiva, al inicio y al final de la ingesta de los nutraceúticos. 
Los individuos, conformados en 4 grupos de 20 cada uno.

Variables: Variable Independiente: Grupos de Tratamiento

Variable Dependiente: Memoria Semántica

Covariables: Edad: 18 a 25 años, Sexo: Masculino y Femenino.

\section{MÉTODO}

Nivel y Tipo de la Investigación: Nivel básico, Tipo experimental y diseño cuasi experimental.

Muestra: Predeterminada - No probabilística Los participantes fueron 80 jóvenes estudiantes entre 18 a 25 años de edad de la Universidad Nacional de la Amazonía Peruana, comensales del Comedor Universitario, conformados en grupos:

Grupo 1: Control o recibieron Placebo (Malto dextrina)

Grupo 2: Recibieron Camu camu

Grupo 3: Recibieron Maca negra

Grupo 4: Recibieron la mezcla, Maca negra-Camu camu

La administración de los extractos y el placebo se realizó durante 6 semanas consecutivas.
Aspectos Éticos

Todoslosmétodos, técnicasyprocedimientos aplicados en la presente investigación están dentro de las normas nacionales e internacionales para proyectos de investigación en humanos.

\section{Instrumentos}

Test de Palabras de Rey (RAVLT, Rey, 1964), permite obtener distintos índices como la amplitud de la memoria a corto plazo, el recuerdo inmediato, el recuerdo demorado, la curva de adquisición a lo largo de los cinco ensayos y la curva de posición serial ampliada.

Análisis de los Datos, se utilizó el programa EXCEL y los datos fueron transferidos al programa STATA versión 10.. Así mismo, se utilizó la prueba binomial (frecuencia esperada en los cambios prepost $\leq 9)$ y la prueba de McNemar para evaluar efectos en la memoria al finalizar el estudio.

Para comparar el total de palabras promedio alcanzado entre los grupos, primero se evaluó la normalidad $y$ homogeneidad de varianzas. Asimismo, se utilizó la técnica de análisis de varianza (ANOVA), y la prueba no paramétrica de Kruskal Wallis; Se utilizó una significancia estadistica de 0.05 para todos los análisis.

\section{III.- RESULTADOS}

Tabla 1.

Descripción de los participantes según grupo de estudio

\begin{tabular}{crrrrr}
\hline Variables & LMaca+camu & 2.Camu camu & 3.Maca & 4.placebo & \multicolumn{1}{c}{ Total } \\
\hline Sexo & & & & & \\
F & $9(45 \%)$ & $9(45 \%)$ & $7(35 \%)$ & $3(15 \%)$ & $28(35 \%)$ \\
M & $11(55 \%)$ & $11(55 \%)$ & $13(65 \%)$ & $17(85 \%)$ & $52(65 \%)$ \\
\hline Edad & & & & \\
Media \pm DS & $21.35 \pm 2.28$ & $21.15 \pm 2.35$ & $21.55 \pm 2.19$ & $20.95 \pm 1.82$ & $21.25 \pm 2.14$ \\
\hline
\end{tabular}


Tabla 2.

\section{Resultados Generales}

Total promedio de palabras alcanzadas en los 10 ensayos según grupo de estudio en el pre y post evaluación

\begin{tabular}{lcccccccc}
\hline \multicolumn{1}{c}{ variable } & $\mathrm{N}^{\circ}$ & media & D.S. & Mediana & Minimo & Máximo & $\mathrm{Zw}$ & $\mathrm{P}$ \\
\hline $\begin{array}{l}\text { maca+camu G1 } \\
\text { Pre test }\end{array}$ & 20 & 13.30 & 1.380 & 13.5 & 10 & 15 & 2.997 & 0.0027 \\
$\begin{array}{l}\text { Post test } \\
\text { camu camu G2 }\end{array}$ & 20 & 14.20 & 1.436 & 15.0 & 9 & 15 & & \\
$\begin{array}{l}\text { Pre test } \\
\text { Post test }\end{array}$ & 20 & 13.55 & 1.932 & 14.0 & 8 & 15 & 1.930 & 0.0537 \\
Maca G3 & 20 & 14.20 & 1.508 & 15.0 & 9 & 15 & & \\
Pre test & 20 & 12.55 & 2.012 & 13.0 & 8 & 15 & 3.063 & $\mathbf{0 . 0 0 2 2}$ \\
Post test & 20 & 13.70 & 1.720 & 14.5 & 10 & 15 & & \\
Placebo G4 & & & & & & & & \\
Pre test & 20 & 13.05 & 1.932 & 14.0 & 9 & 15 & 2.921 & 0.0035 \\
Post test & 20 & 14.05 & 0.999 & 14.0 & 12 & 15 & & \\
\hline
\end{tabular}

En la Tabla 2 podemos apreciar los resultados de los cuatro grupos de estudio. Se evidencia el mayor efecto en la memoria semántica en el Grupo 3 que consumió Maca, exclusivamente.

\section{IV.- DISCUSIÓN}

Respecto al primer objetivo (grupos 2 y 3), en los cuales se mide el efecto de la ingesta de camu camu y maca negra solas, se encuentró un evidente incremento de la memoria semántica en ambos grupos, sin embargo el grupo que hizo ingesta de maca negra sola, presentó una mejora mucho más significativa $(80 \%)$ con una diferencia del $26.2 \%$. Con las consideraciones éticas y de cuidado requeridas hacemos referencia a los resultados hallados en la investigacion realizada en ratas destetadas macho albinos (Ore, y col. 2010), en la cual se usó extracto acuoso de maca negra, para medir la curva de aprendizaje mediante la evaluación acuática de Morris.

En referencia al segundo objetivo, ingesta de la sustancia combinada de maca negra y camu camu, se encontró un incremento significativo en la memoria, sin embargo los resultados encontrados se reducen en comparación con los resultados obtenidos solamente con maca negra.

Con respecto al tercer objetivo especifico, las curvas de aprendizaje como efecto de la ingesta sola o combinada de los nutracéuticos son significativoas predominando el de la maca negra (de 3 en el pre test a 10 en el post test). También se encuentra en el grupo control un incremento reducido, que podria interpretarse como que variables externas del contexto (no controladas) han permitido el incremento encontrado, que también pueden haber contribuido en los grupos experimentales. .

Es importante señalar, que en las 2 comparaciones que se le hicieron con investigaciones realizadas en animales y el uso de la maca negra, las ratas utilizadas fueron de alrededor de quince días de edad, lo que correspondería a la edad proporcional de 18 años aproximadamente en humanos; y con un tiempo de ingesta de 15 días que equivaldria a 6 meses en humanos, por lo que la explicación de estos resultados debe ser tomada con mucho cuidado para la extrapolación en humanos. 


\section{V.- CONCLUSIÓN}

Los efectos de los nutracéuticos maca negra y camu camu, solos y combinados, son evidentes en el incremento de la curva de aprendizaje al evaluar la memoria semántica, siendo mayor con la ingesta de la maca negra exclusivamente.

\section{VI.- RECOMENDACIONES}

Se recomienda efectuar investigaciones con población de distintos grupos etarios, incrementando la ingesta de maca negro y camu camu.

\section{VII.- REFERENCIAS BIBLIOGRÁFICAS}

Aguilar L, Espinoza G, Oruro E, León R. (2013) Breves consideraciónes sobre el papel del sueño en el aprendizaje. Aletheia 1 (1): 113-121.

Akachi T, Shiina Y, Kawaguchi T, Kawagishi H, Morita T, Sugiyama K. (2010) 1-metbylmalate from camu-camu (Myrciaria dubia) suppressed D-galactosamine-induced liver injury in rats. Biosci. Biotechnol. Biochem. 23;74(3):5738. Epub.

Bayona, C. (1999) E/ Camu Camu, fuente de vitamina C. Servicio Informativo Iberoamericano. Mayo 1999 (2). http://www.oei.org.co/sii/ entrega15/art06.htm\#aa

Bradfield R. B., Roca A.(1964) Camu camu - A fruit bigh in ascorbic acid. J Am. Diet Assoc. pag 2830.

Canales, M., Aguilar, J., Prada, A., Marcelo, A., Huamán, C., \& Carbajal, L. (2000). Evaluación nutricional de lipidium meyenii (MACA) en ratones albinos $y$ st descendencia. Archivos. Latinoamericanos de Nutrición, 50(2), 12633.

Campodónico, C. (2009) La ingesta de Lepidium Peruvian y el desarrollo de las células de la linea espermatogénica e intersticial de Leyding en el testiculo de ratas jóvenes boltzman. Odontología Sanmarquina; 12(1): 10-12
Chirinos R, Galarza J, Betalleluz-Pallardel I, Pedreschi R, Campos D. (2010) Antioxidant compounds and antioxidant capacity of peruvian camu camu (Myrciaria dubia (H.B.K.) Mc Vaugb) fruit at different naturity stages. Food chemistry 120, pp $1019-1024$.

Clément C, Kneubühler J, Urwyler A, Witschi U, Kreuzer M. (2010) Effect of maca supplementation on bovine sperm quantity and quality followed over two spermatogenic gycles. Theriogenology. 74(2):173-83. Epub 2010 May 10.

Collazos Ch C; P.L. White; H. S. White; E. Viñas T.; E. Alvistur J.; R. Urquieta A.; J. Vásquez, C. Díaz T.; A. Quiroz M.; A. Roca N,; D. M. Hegted y R. B. Bradfield. (1957). La composición de los alimentos peruanos. Anales de la Facultad de Medicina, Tomo XL-N ${ }^{\circ} 1$, Lima.

Dabrowski K, Jun-Lee K, Richard J, Abiado M G, Alcántara B F, Tello S, Palacios M E. (2004) Studies on reproduction and larval rearing of Amazonian fish. en R. Harris, I. Courtey and H. Egua (Editors), Twenty-First Annual Technical Report. Aquaculture CRSP, Oregon. State University, Corvalles, Oregon, pp. 85-92.

Genovese I M, Da Silva P M, De Souza A.E, Schmidt G and Lajolo F M. (2008) Bioactive Compounds and Antioxidant Capacity of Exotic Fruits and Commercial Frozen Pulps from Brazil. Food Science and Technology International. 14: 207-214.

Gonzales C, Cárdenas-Valencia I, Leiva-Revilla J, Anza-Ramirez C, Rubio J, Gonzales GF. (2010) Effects of different varieties of Maca (Lepidium meyenii) on bone structure in ovariectomized rats.Forsch Komplementmed. 17(3):137-43. Epub 2010 Jun 16.

Gonzales C, Leiva-Revilla J, Rubio J, Gasco M, Gonzales GF. (2011) Effect of red maca (Lepidium meyenii) on prostate zinc levels in rats with testosterone-induced prostatic 
byperplasia. Andrologia. doi: 10.1111/j.14390272.2011.01190.x. [Epub ahead of print] Gonzales C, Rubio J, Gasco M, Nieto J, Yucra S, Gonzales GF. (2006) Effect of short-term and long-term treatments with three ecotypes of Lepidium meyenii (MACA) on spermatogenesis in rats. J Ethnopharmacol. 103(3):448-54. Epub 2005 Sep 19.

Gonzales GF, Cordova A, Gonzales C, Chung A, Vega K, Villena A. (2001) Lepidium meyenii (Maca) improved semen parameters in adult men. Asian J Androl. 3(4):301-3.

Gonzales GF, Gasco M, Córdova A, Chung A, Rubio J, Villegas L. (2004) Effect of Lepidium meyenii (Maca) on spermatogenesis in male rats. acutely exposed to bigh altitude $(4340 \mathrm{~m})$. J Endocrinol. 180(1):87-95.

Gonzales GF, Gonzales C, Gonzales-Castañeda C. (2009) Lepidium meyenii (Maca): a plant fram the bighlands of Peru-from tradition to science. Forsch Komplementmed. 16(6):373-80. Epub 2009 Dec 16. Review.

Gonzales GF, Nieto J, Rubio J, Gasco M. (2006) Effect of Black maca (Lepidium meyenii) on one spermatogenic gycle in rats. Andrologia. 38(5):166-72.

Gonzales GF, Rubio J, Chung A, Gasco M, Villegas L. (2003) Effect of alcobolic extract of Lepidium meyenii (Maca) on testicular function in male rats. Asian J Androl. 5(4):349-52.

Inoue T, Komoda H, Uchida T, Node K. (2008) Tropical fruit camu-camu (Myrciaria dubia) bas anti-oxidative and anti-inflammatory properties. J Cardiol. 52(2):127-32. Epub 2008 Jul 29.

Justi KC, Visentainer JV, Evelázio de Souza N, Matsushita M. (2000) Nutritional composition and vitamin $C$ stability in stored camu-camu (Myrciaria dubia) pulp. Arch Latinoam Nutr. 50(4):405-8.

Klinar B. S., Chanllio I. J y Chang C. A. (2009) Evaluación comparativa de contenido de vitamina $C$ en frutos de camu camu Myrciaria dubia (H. B. \& K.) Mc Vaugb, Maracuya Passiffora edulis Simsy
Cocona Solanum sessilifforum Dunal. FITOICA Revista Científica Año 4- $\mathrm{N}^{\circ} 3$.

Luria, Y. (1979) El cerebro en acción. Fontanella. Madrid. Ministerio de Agricultura / INRENA (2000) Programa Nacional de Camu Camu 2000 - 2020. Muñoz J. A M. (2007). Evaluación de la capacidad antioxidante y contenido de compuestos fenólicos en recursos vegetales promisorios. Rev. Soc. Quim. Perú, 73, No 3 (142-149).

Muñoz Jauregui A.M, Ramos-Escudero F, Alvarado-Ortíz U C, Castañeda C.B, Lizaraso C.F. (2009) Evaluación de compuestos con actividad biológica en cáscara de camu camu (Myrciaria dubia), guinda (Prunus serotina), Tomate de árbol (Cyphomandra betacea) y Carambola (Averrboa carambola. L) cultivadas en Perí. Rev. Soc Quím Perú. 75 (4).

Ramos Ll. E, Castañeda C.B, Ibáñez V.L. (2008) Evaluación de la capacidad antioxidante de plantas medicinales peruanas nativas e introducidas. Rev. Acad. Peru Salud 15 (1).

Reyes G. M., Gómez-Sánchez P I, Espinoza B.C,Bravo R F y Ganoza M L. (2009) Tablas Perianas de Composición de Alimentos. Centro Nacional de Alimentación y Nutrición. 8va. Ed. Ministerio de Salud-INS. Lima, 64p.

Reynertson K.A; Yang H. (2008) Quantitative analysis of antiradical phenol constituents from fourteen edible Myrtaceae fruits. Food Chem; 109 (4); 883-890

Rubio J, Dang H, Gong M, Liu X, Chen SL, Gonzales GF. (2007) Aqueous and bydroalcobolic extracts of Black Maca (Lepidium meyenii) improve scopolamine-indwced memory impairment in mice. Food Chem Toxicol; 45(10):1882-90. Epub 2007 Apr 20.

Rubio J, Riqueros MI, Gasco M, Yucra S, Miranda S, Gonzales GF. (2006) Lepidium meyenii (Maca) reversed the lead acetate induced -- damage on reproductive function in male rats. Food Chem Toxicol; 44(7):1114-22. Epub 2006 Feb 28.

Saldaña, C.; Córdova, O. y Vargas, F. (2006). Utilización de Lepidium Perunianum Maca, como 
medio de cultivo para el crecimiento de Trypanosoma Cruzi. Revista Peruana de Medicina Experimental y Salud Pública 23(2) pp. 137 140. Disponible en:

<http://www.scielo.org.pe/scielo. php?script $=$ sci_arttext\&pid $=\$$ 1726 $46342006000200010 \& \operatorname{lng}=\mathrm{es} \& \mathrm{nrm}=\mathrm{iso}>$. ISSN 1726-4634.

Sotero S V, Silva D L, Garcia D, Iman C S. (2009) Evaluación de la actividad antioxidante de la pulpa, cáscara y semilla del fruto del camu camu (Myrciaria dubia (H. B. K.). Rev. Soc Quím Perú. 75 (3). 293-299.

Stone M, Ibarra A, Roller M, Zangara A, Stevenson E. (2000) A pilot investigation into the effect of maca supplementation on physical activity and sexual desire in sportsmen. J Ethnopharmacol; 126(3):574-6. Epub 2009 Sep 23.

Villanueva-Tiburcio, J.E; Cardozo-Hoyos, L.A; Asquieri, E.R. (2010) Antocianinas, ac.ascórbicopolifenoles totales y actividad antioxidante en la cáscara de camu camu (Myrciaria dubia (H. B. K.) Mc Vaugh. Cienc. Tecnol. Aliment., Campinas, 30 (supl. 1): 151-160.

Wang Z, Yang J, Wang G, Bian L. (2009). Influence of Lepidium meyemii walp on lipid and bone mass in ovariectomized rats. Wei Sheng Yan Jiu; $38(4): 420-2,425$.

Zanatta C. F., Cuevas E., Bobbio F. O, Winterhalter P. and Mercante A.Z. (2005) Determination of Anthocyanins from camu camu (Myrciaria dubia) by HPLC - PDA, HPLC - MS and NMR. J. Agric. Food Chem, 53; 9531-9535.

Zanatta, C. F., Mercadante, A. Z. (2007) Carotenoid composition from the Brasilian tropical fruit camt camu (Myrciaria dubia) Food Chemistry 101. 1543-1549.

Zenico T, Cicero AF, Valmorri L, Mercuriali M, Bercovich E. (2009) Subjective effects of Lepidium meyenii (Maca) extract on well-being and sexual performances in patients with mild erectile dysfunction: a randomised, double-blind clinical trial. Andrologia; 41(2):95-9. 
Recibido: 18-08-2016 Aceptado: 31-08-2016

Palabras clave: vNeurociencia, Sueño, Somnolencia, Apnea. Aprendizaje.

Keywords: Neuroscience, sleep, sleepiness, apnea. Learning.

byal12@hotmail.com Universidad San Carlos Guatemala Elena Nelly Garcia-Lopez Hernandez nelly.garcialopezh@gmail.com Universidad de Alcalá - España Carmen Estela Montenegro S. carmen.montenegro@upch.pe UPCH

Cesar Malpartida Porras cesar.malpartida@yahoo.es

UPCH

María Lucia Baquerizo S. malubaq@neurocienciaperu.org $\mathrm{UPCH}$

Cinthya Vanessa Minaya vminaya@neurocienciaperu.org UPCH

Luis Aguilar luis.aguilar@upch.pe UPCH - UNIFE

\section{Investigación sobre el sueño en estudiantes universitarios de Guatemala y el Salvador: Aproximaciones y características}

\author{
Byron Alvizures $^{1}$, Nelly Garcia ${ }^{2}$, Estela Montenegro ${ }^{3}$, \\ Cesar Malpartida ${ }^{4}$, Marilia Baquerizo ${ }^{4}$, Vanessa Minaya ${ }^{4}$, \\ Luis Aguilar ${ }^{3,4}$,
}

RESUMEN. Durante la neurofisiología, la calidad de vida tiene relación con los ritmos circadianos, espetando las horas de vigilia (despierto) como las horas de sueño (dormir). Un buen control horario de estas variables dan una buena calidad de vida en general, donde los aportes en el aprendizaje se ven comprometidos. En el presente trabajo, se realizó el estudio de calidad de sueño, a través de la escala de Epworth y Berlin, a un grupo de estudiantes de Medicina y Odontología de una universidad de Guatemala y otra de El Salvador. La aplicación de los instrumentos de manera transversal revelo que La probabilidad de padecer apnea se presentan en solteros mas que en casados. El $6 \%$ presenta apnea y en una proporción de 2 varones/1 mujer. $13 \%$ presentan somnolencia diurna alta, y es indistinto a varones o mujeres. Los alumnos evaluados, por su estilo de vida, están más proclives a padecer de un trastorno de sueño, específicamente Apnea y somnolencia diurna. Las universidades $\mathrm{e}$ instituciones de salud pública deben tomar acciones para prevenir $y / o$ tratar los trastornos de sueño, específicamente apnea y somnolencia diurna, con el afán de disminuir los problemas de aprendizaje y mejorar la calidad académica del alumno.

\begin{abstract}
The neurophysiology, quality of life is related to circadian rhythms, broaching waking hours (awake) as hours of sleep (sleep). A good time control of these variables provide a good quality of life in general, where contributions in learning are compromised. In this paper, the quality of sleep study was conducted by the Epworth scale and Berlin, a group of students of Medicine and Dentistry of a university in Guatemala and one in El Salvador. Applying cross-cutting instruments revealed that the probability of developing apnea are presented in more singles than married. $6 \%$ apneic and a 2 males / 1 female. $13 \%$ have high daytime sleepiness, and is indifferent to men or women. Students evaluated their lifestyle, are more likely to suffer from a sleep disorder, specifically apnea and daytime sleepiness. Universities and colleges of public health must take action to prevent and / or treat sleep disorders, specifically apnea and daytime sleepiness, in an effort to reduce the problems of learning and improving the academic quality of the student.
\end{abstract}




\section{I.- INTRODUCCION}

$\mathrm{L}$

os trastornos del sueño por diversas causas presentan una alta prevalencia en la población general (35-40\%). Entre estos trastornos destacan por su frecuencia el SAHOS (síndrome de apneas/hipoapneas obstructiva del sueño), que en estudios internacionales, afecta a 1 de cada 5 adultos en su presentación leve y a 1 de cada 15 en su presentación moderada a severa y el insomnio, que en series internacionales afecta a $5-34 \%$ de la población

\section{Apnea obstructiva, una pesadilla en la noche}

No todos los roncadores pueden tener apnea de sueño. Pero la totalidad de quienes padecen esa dolencia, roncan. Acqui sus caracteristicas.

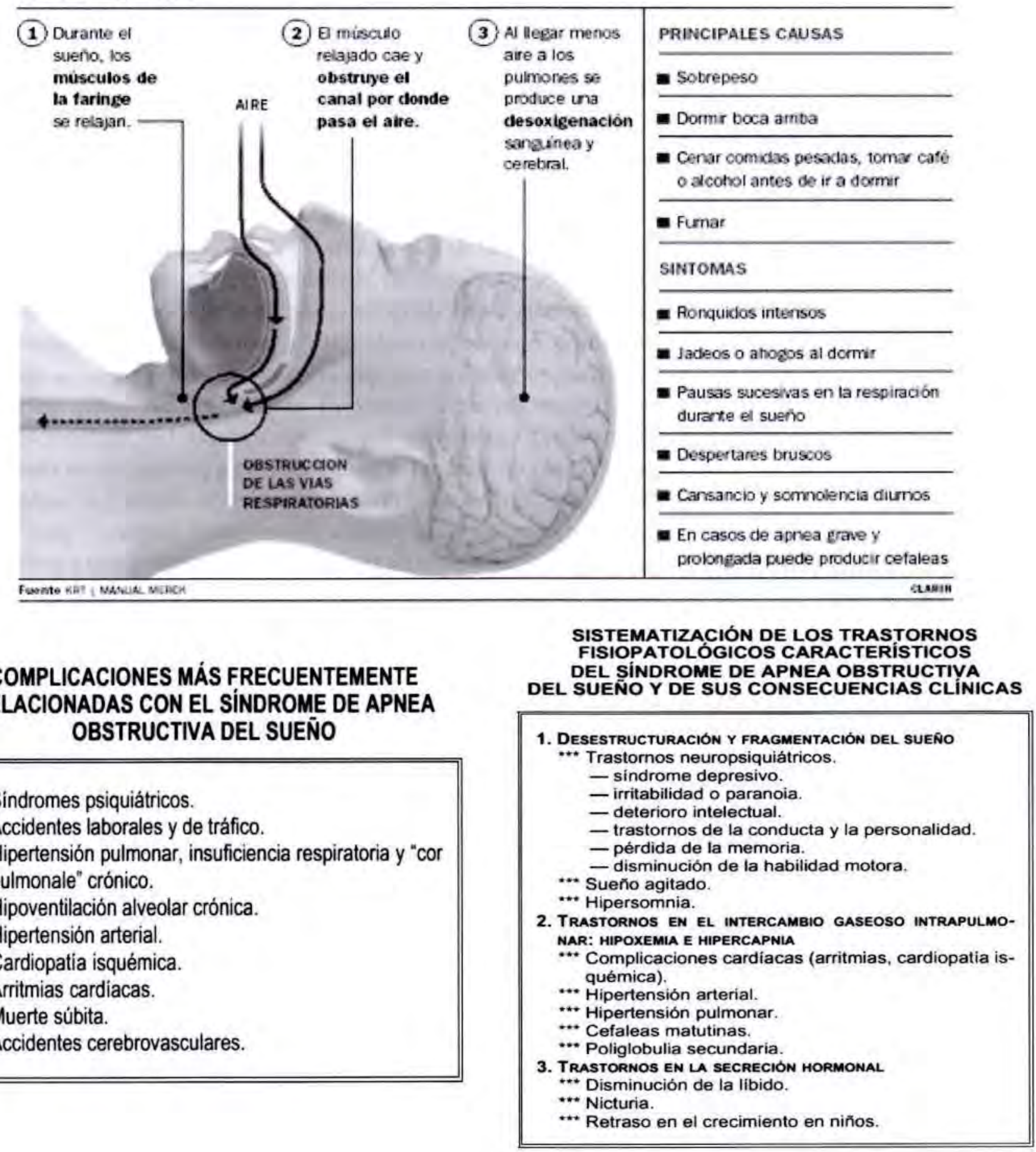




\section{II.- HIPOTESIS}

Si conoces la importancia del sueño en la calidad de vida, entonces la población no debe padeceré de somnolencia ni apnea en el sueño.

\section{IV.- OBJETIVOS}

\section{OBJETIVO GENERAL}

Determinar si los encuestados conocen y aplican la importancia de la neurofisiología del sueño en la calidad de vida de las personas.

\section{OBJETIVOS ESPECIFICOS}

Si la somnolencia afecta la vida diaria de los estudiantes encuestados

Si la Apnea de sueño afecta la vida diaria de los estudiantes encuestados

Si hay diferencia en la presencia de somnolencia y apnea en el sueño a nivel de genero en los estudiantes encuestados.

\section{V.-CARACTERÍSTICAS MUESTRALES DE LA INVESTIGACIÓN}

Poblacion estudiada: 334 individuos.

Se aprecia una edad promedio de 22.6 años, básicamente estudiantes.

$\mathrm{Y}$ las edades extremas superiores de 50 a 60 años corresponden a los docentes y médicos (07 casos).

\begin{tabular}{|l|}
\hline LUGAR LABORA \\
\hline Quetzaltenango \\
\hline Huehuetenango \\
\hline San Marcos \\
\hline Totonicapan \\
\hline
\end{tabular}

\begin{tabular}{|l|}
\hline OCUPACIÓN \\
\hline Docente \\
\hline Estudiante \\
\hline Médico Cirujano \\
\hline
\end{tabular}

\section{VI.- METODOLOGIA}

Se aplicaron los test:
1.- CUESTIONARIO DE BERLIN: Síndrome de apneas/hipoapneas obstructiva del sueño

2.- ESCALA DE EPWORTH MODIFICADA: Somnolencia diurna

Los test 1 y 2 se aplicaron de manera transversal en estudiantes de Medicina, Odontología, médicos y Docentes de las Universidades de San Carlos de Guatemala y San Salvador de el Salvador. Corte tranversal.

\section{VII.-RESULTADOS Y DISCUSION}

CUESTIONARIO DE BERLIN: Síndrome de apneas/hipoapneas obstructiva del sueño

El $94.3 \%$ no presentan probabilidad de tener SAHOS, es decir, que solo $5.7 \%$ de la muestra si presente probabilidad de tener SAHOS.

\section{SEGÚN SEXO}

De las personas que presentan probabilidad significativa de SAHOS, el $31.6 \%$ es de sexo femenino y el $68.4 \%$ es de sexo masculino.

Los varones son más propensos a tener SAHOS que las mujeres $(2 / 1)$.

ESTADO CIVIL

El $92.8 \%$ de la muestra es soltero

Al observar el cuadro de probabilidad de SAHOS según estado civil, se puede observar que de las personas que presentan probabilidad de SAHOS, el $89.5 \%$ es soltero y solo el $10.5 \%$ casado.

\section{OCUPACIÓN}

Se observa que el $100 \%$ de personas que presentan probabilidad de SAHOS, es estudiante.

ESCALA DE EPWORTH MODIFICADA: Somnolencia diurna

En la muestra, solo el $12.6 \%$ presenta somnolencia diurna alta. Este grupo, tiene en promedio 22.07 años

\section{SEGÚN SEXO}

El $45.2 \%$ de quienes presentan somnolencia diurna alta son mujeres y el $54.8 \%$ son varones. No hay diferencia significativa $(1 / 1)$ 


\section{SEGÚN OCUPACIÓN}

El $100 \%$ de estudiantes, presentan somnolencia diurna alta.

De ello se corresponde con que también el $100 \%$ de personas que tienen probabilidad de SAHOS sean estudiantes.

\section{RELACIÓN DE BERLIN Y EPWORD}

Al relacionar los resultados de ambas pruebas, Se interpretaría que no hay una relación directa entre la escala EPWORTH (Somnolencia) y APNEA. Es decir, que a mayor valor en EPWORTH (somnolencia) no necesariamente aumenta el valor de APNEA

\section{VIII.- CONCLUSIONES}

- La probabilidad de padecer SAHOS se presentan en solteros mas que en casados en una proporción de $9 / 1$.

- 6 de cada 100 alumnos de la muestra presentan probabilidad de tener SAHOS, en una proporción de 2 varones/ 1 mujer.

- 13 de cada 100 alumnos presentan somnolencia diurna alta, y es indistinto a varones o mujeres.

- Los alumnos evaluados, por su estilo de vida, están más proclives a padecer de un trastorno de sueño, específicamente SAHOS y somnolencia diurna.

\section{IX.- RECOMENDACIONES}

- Se podría investigar más trastornos de sueño dentro de una muestra de estudiantes, incluyendo otras variables.

- Las universidades e instituciones de salud pública deben tomar acciones para prevenir y/o tratar los trastornos de sueño, específicamente SAHOS y somnolencia diurna, con el afán de disminuir los problemas de aprendizaje y mejorar la calidad academica del alumno.

\section{X.- REFERENCIAS BIBLIOGRÁFICAS}

Aguilar L. 2015. Bases Conceptuales de las Neurociencias. En Cognicion, Neurociencia y Aprendizaje. El adolescente en la educación superior. : Pease. M, Figallo F, Ysla L. Fondo Editorial PUCP. Lima.Peru.

Gallego J. 2013. Calidad de Sueño y Somnolencia diurna en estudiantes de enfermería: Estudio de prevalencia. Tesis Doctoral. Universidad Catolica San Antonio. Murcia. España.

Sierra J, Jimenes-Navarro C, Domingo J. 2002. Calidad del sueño en estudiantes universitarios: Importancia de la Higiene de sueño. Salud Mental, Vol. 25 No. 6. Diciembre.

Castillo J, Araya F, Montecino L, Torres C, Oporto s, Bustamante G, Aranda w. 2008. Aplicación de un cuestionario de sueño y la escala de somnolencia de Epworth en un centro de Salud Familiar. Rev Chil Neuro-psiquiat; 46(3):182-191.

Borquez P. 2011. Calidad de Sueño, somnolencia diurna y salud autopercibida en estudiantes universitarios. EUREKA. Asuncion (Paraguay) $8(1): 80-91$.

Salas A. 2011. Calidad de Sueño y su relación con las estrategias de afrontamiento y cronotipo de estudiantes de Odontologia. Tesis Doctoral. Universidad Autonoma de Madrid. Madrid. España.

Moreno M. 2012. Calidad de Sueño y Salud Mental en estudiantes de farmacia de la Universidad de los Andes, Merida-Venezuela. Tesis Doctoral. Universidad Autonoma de Madrid. Madrid. España.

Faubel R. 2008. El impacto de la duración habitual del sueño sobre la obesidad, la presión arterial, la calidad de vida y la función cognitiva de los ancianos españoles. Tesis Doctoral. Universidad Autonoma de Madrid. Madrid. España.

Escobar f, Oscar j, Eslava j. 2006. Calidad de Sueño en mujeres victimas de asalto sexual en Bogota. Rev Fac Med Univ Nac Colomb. Vol 54 No.1. 